\title{
Semi-parametric Analysis of Dynamic Contrast-Enhanced MRI Using Bayesian P-Splines
}

\author{
Volker J. Schmid ${ }^{1}$, Brandon Whitcher ${ }^{2}$, and Guang-Zhong Yang ${ }^{1}$ \\ ${ }^{1}$ Institute for Biomedical Engineering, Imperial College, South Kensington, London \\ SW7 2AZ, United Kingdom \\ $\{$ v.schmid, g.z.yang\}@imperial.ac.uk \\ 2 Translational Medicine \& Genetics, GlaxoSmithKline, Greenford Road, Greenford \\ UB6 0HE, Middlesex, United Kingdom \\ brandon.j.whitcher@gsk. com
}

\begin{abstract}
Current approaches to quantitative analysis of DCE-MRI with non-linear models involve the convolution of an arterial input function (AIF) with the contrast agent concentration at a voxel or regional level. Full quantification provides meaningful biological parameters but is complicated by the issues related to convergence, (de-)convolution of the AIF, and goodness of fit. To overcome these problems, this paper presents a penalized spline smoothing approach to model the data in a semi-parametric way. With this method, the AIF is convolved with a set of B-splines to produce the design matrix, and modeling of the resulting deconvolved biological parameters is obtained in a way that is similar to the parametric models. Further kinetic parameters are obtained by fitting a non-linear model to the estimated response function and detailed validation of the method, both with simulated and in vivo data is provided.
\end{abstract}

\section{Introduction}

Dynamic contrast-enhanced magnetic resonance imaging (DCE-MRI) has become an important source of information to aid cancer diagnosis. After the administration of a contrast agent, such as Gadolinium diethylenetriaminepentaacetic acid (Gd-DTPA), a dynamic sequence of MR scans is acquired, typically using $T_{1}$-weighted sequences for assessing the reduction in $T_{1}$ relaxation time caused by the contrast agent. The contrast concentration may be estimated from the observed signal intensity using proton density-weighted images after calibration [1. Quantitative methods based on pharmacokinetic (PK) models are then used to fit a non-linear function to the estimated contrast agent concentration. These models are usually derived from the solution to a system of linear differential equations 2 .

A two-compartment model describing flow between plasma (or vascular space) and extra-vascular extra-cellular space (EES) is commonly used. Mathematically, this can be described as

$$
C_{t}(t)=C_{p}(t) \otimes K^{\text {trans }} \exp \left(-k_{\mathrm{ep}} t\right) .
$$


where $C_{t}$ is the concentration of contrast agent in the tissue at time $t, C_{p}$ the arterial input function (AIF) and the kinetic parameters of interest are $K^{\text {trans }}$ and $k_{\mathrm{ep}}$. If the AIF is given in parametric form, the convolution in (11) may be written in an analytical form and the contrast agent concentration can be estimated by using standard non-linear regression methods 3 .

Recently, non-parametric or model-free techniques for DCE-MRI have received extensive attention. These include the use of neural networks 4 for tissue classification, and semi-parametric methods for kinetic modeling of dynamic PET imaging [5]. Jerosch-Herold et al. 6] have proposed a model-free approach for myocardial perfusion quantification, based on a B-spline polynomial representation of the response function. The formulation of this problem, however, is ill-conditioned and a first-order difference penalty was imposed.

Penalty splines, or P-splines, represent another popular approach for semiparametric modeling [7].The underlying function is approximated by a linear combination of a relatively large number of B-spline basis functions, where smoothness is ensured by a penalty based on $k$-th order differences of the parameter vector. For selecting the penalty weight (or smoothing parameter) $\lambda$ cross validation can be used. Bayesian versions of $\mathrm{P}$-splines estimate $\lambda$ jointly with the model and allow for varying weights; i.e. adaptive smoothing 8 .

In practice, an adaptive approach to modeling the contrast agent concentration in DCE-MRI is preferred since the observed time series vary rapidly in the first two minutes after bolus injection of the contrast agent. The purpose of this paper is to apply Bayesian $\mathrm{P}$-spline regression to the estimated contrast agent concentration, proposing an ad-hoc estimator for plasma flow, derived directly from the response function. Further kinetic parameters are obtained by fitting a non-linear model to the estimated response function. Detailed validation of the method both with simulated data and clinical cases of breast tumor is provided.

\section{Theory and Methods}

\section{$2.1 \quad$ Theoretical Background}

The time series of measured contrast agent concentration $C_{t}(t)$ is the convolution of the arterial input $C_{p}$ and the response function $f$, such that

$$
C_{t}(t)=C_{p}(t) \otimes f(t)=\int_{0}^{t} C_{p}(t-u) f(u) d u .
$$

The concentration is measured at discrete time points $\tau_{1}, \ldots, \tau_{n}$. The AIF may be measured (or estimated) at a different resolution $t_{1}, \ldots, t_{T}$, or a standardized function as e.g. proposed by Tofts and Kermode [9] may be used. The discretized form of (2) is given by

$$
C_{t}\left(\tau_{i}\right)=\sum_{j=1}^{T} C_{p}\left(\tau_{i}-t_{j}\right) f\left(t_{j}\right) \Delta t=\sum_{j=1}^{T} A_{i j} f\left(t_{j}\right),
$$


where $\Delta t$ represents the sampling interval of the AIF. The $T \times n$ matrix $\boldsymbol{A}$ may be interpreted as a convolution operator and is defined via

$$
A_{i j}= \begin{cases}C_{p}\left(\tau_{n_{i}-j+1}\right) \Delta t & \text { if } t_{i}<\tau_{j} \\ 0 & \text { else, }\end{cases}
$$

where $n_{i}$ is the maximum index $j$ for which $t_{j} \leq \tau_{i}$ holds. To improve numerical stability, a B-spline representation for the response function is used in this study such that

$$
f(t)=\sum_{j=1}^{p} \beta_{j} B_{j t},
$$

where $\boldsymbol{B}$ is the $n \times p$ design matrix of $k$ th order B-splines with knots $s_{1}, \ldots, s_{p+k}$. More knots are placed in the first two minutes (about every 3-5 seconds) and fewer afterwards (about every 20-30 seconds) with a total of approximately 4050 knots. In vector notation $\boldsymbol{f}=\left(f\left(t_{1}\right), \ldots, f\left(t_{T}\right)\right)^{\prime}$ and (5) may be expressed via $\boldsymbol{f}=\boldsymbol{B} \boldsymbol{\beta}$. When we put this into (3), we get

$$
C_{t}=A f=A B \beta=D \beta,
$$

with $\boldsymbol{D}=\boldsymbol{A} \boldsymbol{B}$ a $T \times p$ design matrix, which is the (discrete) convolution of the AIF with the B-spline polynomials.

\subsection{Bayesian Model}

A fully Bayesian hierarchical model is used to fit the proposed semi-parametric model. Following the notation in the previous section, the corresponding data model in each voxel becomes

$$
C_{t}(t)=\boldsymbol{D}_{t} \boldsymbol{\beta}+\epsilon_{t} \text { for each } t,
$$

where $\boldsymbol{D}_{t}$ denotes the $t$ th row of $\boldsymbol{D}$, with $\boldsymbol{D}=\boldsymbol{A} \boldsymbol{B}$.

Although the system of equations is linear in $\boldsymbol{\beta}$, (7) may still be numerically unstable. A stochastic constraint on $\boldsymbol{\beta}$ is introduced in the process model using a second-order random walk prior, to aquire a reasonably smooth representation of the response function. That is, starting from the third coefficient, each coefficient is a priori assumed to be the linear continuation of the last two coefficients

$$
\beta_{i}=2 \beta_{i-1}-\beta_{i-2}+e_{i} \quad \text { for } i=3, \ldots, p .
$$

The inverse variance (precision) $\delta_{i}$ of the error term $e_{i}$ in (8) determines the smoothness of the spline. A non-informative hyperprior is proposed, $e_{t} \sim$ $\mathrm{N}\left(0,1 / \delta_{t}\right), \delta_{t} \sim \mathrm{Ga}\left(10^{-5}, 10^{-5}\right)$, where Ga denotes the Gamma distribution. As the response function exhibits a sharp increase and a sharp decrease right at the beginning of the dynamic series, the smoothness parameter is estimated adaptively, i.e., the precision $\delta_{t}$ is allowed to vary over time.

For the observation error $\epsilon$ we assume a priori a Normal distribution with unknown variance, $\epsilon_{t} \sim \mathrm{N}(0,1 / \kappa), \kappa \sim \mathrm{Ga}(1,0.05)$. Bayes' theorem is applied to calculate the posterior distribution of the parameter vector. We use a Markov chain Monte Carlo (MCMC) algorithm to obtain samples from the posterior distribution [10]. 


\subsection{Obtaining Kinetic Parameters}

In order to obtain kinetic parameters, the mean plasma flow $F_{p}$ is estimated via the maximum of the response function. This is equivalent to the suggestions of Jerosch-Herold et al. [6], who use the maximum of the response function to estimate the maximum blood flow in myocardial perfusion.

To obtain additional kinetic parameters, a non-linear model is fitted to the response function. St. Lawrence and Lee 11 have proposed a step function in their adiabatic approximation to the tissue homogeneity model. In practice, a step function is highly unlikely to be observed especially in the smoothing framework presented here, so a linear approximation is used instead given by

$$
H(t)= \begin{cases}1-t(1-E) / \tau & \text { for } 0 \leq t<\tau ; \\ E \exp \left[-(t-\tau) E F_{p} / v_{e}\right] & \text { for } t \geq \tau\end{cases}
$$

and $f(t)=F_{p} \cdot H(t)$. Here $\tau=2 T_{C}$, the transit time through the capillary, $E$ is the extraction fraction of the tracer that is extracted into the EES during a single capillary transit and $v_{e}$ is the volume fraction of EES.

It is worth noting that the Bayesian methodology in Section 2.2 provides not just one response function, but a distribution on the space of response functions. By fitting (9) to each response function in the sample we obtain a distribution of the estimated parameters. The median of the posterior distributions is used to estimate the parameters, the estimation error can be estimated via the standard errors of the samples and intervals can be constructed from quantiles of the posterior distributions.

\section{Results}

\subsection{Validation on Simulated Data}

To assess the underlying accuracy of the method, the proposed method is applied to simulated data provided by David Buckley [12] with known kinetic parameters. The data was simulated using MMID4, part of a software made available by the National Simulation Resource, Dept. of Bioengineering, University of Washington (http://www.nsr.bioeng.washington.edu). We use the first series from [12, which was designed to be representative of data acquired from a breast tumor.

As a reference parametric model, an extended version of the modified Kety model is used

$$
C_{t}(t)=v_{p} C_{p}(t)+K^{\mathrm{trans}} \int_{0}^{t} C_{p}(u) \exp \left[-k_{\mathrm{ep}}(t-u)\right] d u,
$$

which adds a vascular term $v_{p}$ (plasma volume fraction) to the standard model, see (11). Note, that $K^{\text {trans }}=E F_{p}, k_{\mathrm{ep}}=K^{\text {trans }} / v_{e}$ and $v_{p}=T_{c} / F_{p}$.

As one would expect, the goodness of fit is much better with the semiparametric technique than with the parametric model. The mean of the residual 
(a) $K^{\text {trans }}$

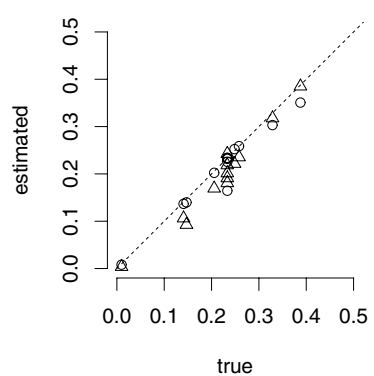

(b) $k_{\mathrm{ep}}$

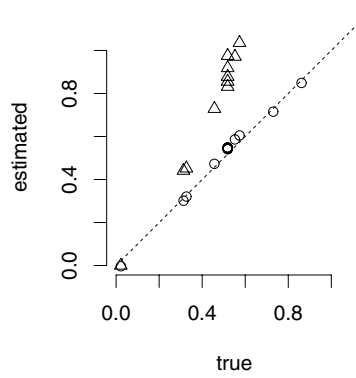

(c) $v_{p}$

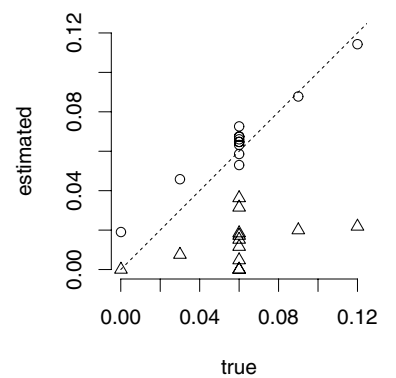

Fig. 1. Scatter plot of parameter estimates vs. true values. Estimates of the parameters (a) $K^{\text {trans }}$, (b) $k_{\text {ep }}$ and (c) $v_{p}$. Circles represent the results using the semi-parametric method, triangles the results using the parametric technique.

sums of squares for the semi-parametric method, over all 13 experiments, was $1.13 \cdot 10^{-3}$ (ranging from $1.89 \cdot 10^{-4}$ to $2.13 \cdot 10^{-3}$ ). This implies that the data was nearly interpolated by this model. The mean of the residual sums of squares for the parametric model was 0.661 (ranging from 0.198 to 1.520 ).

Fig. 1 shows the parameter estimates for both methods as a function of the true values. The parameter $K^{\text {trans }}$ is underestimated with the parametric model (in average by $17.7 \%$, consistent with previously reported results, [12]), whereas the semi-parametric technique is doing much better (mean deviation from true values is $6.2 \%$ ). The parameter $k_{\mathrm{ep}}$ is strongly overestimated with the parametric method (between $38 \%$ and 100\%), as $v_{p}$ is underestimated (by $39 \%$ up to $100 \%)$. With the semi-parametric approach, $k_{\text {ep }}$ is slightly overestimated only (by $1.5 \%$ to $6.2 \%$ ), whereas $v_{p}$ is estimated well at 11 out of the 13 experiments (with error of $2.5 \%$ to $21 \%$ ), whereas two values show a less good fit ( $52 \%$ error and a value of 0.18 instead of $\left.10^{-4}\right)$. Overall, the parameters obtained from the semi-parametric approach fit much better to the ground truth.

The maximum response function (MRF) can be used to estimate $F_{p}$. The values of the MRF and the estimation of $F_{p}$ via non-linear fitting from the estimated response function are nearly equivalent, with a correlation of $99.94 \%$. Fig. 2(a) shows both the non-linear fitting from the response function and the MRF plotted against the true values. With the proposed Bayesian technique, not only are the parameters estimated, but estimations of the error or interval estimators are also available. Fig. 2(b) gives 95\% credibile intervals for $K^{\text {trans }}$. When the point estimate is relatively far away from the true value (Exps. 6, 12 and 13) the intervals are wide and cover the true value, indicating that the technique provides important information about both the accuracy and precision of its estimates. 
(a) $F_{p}$

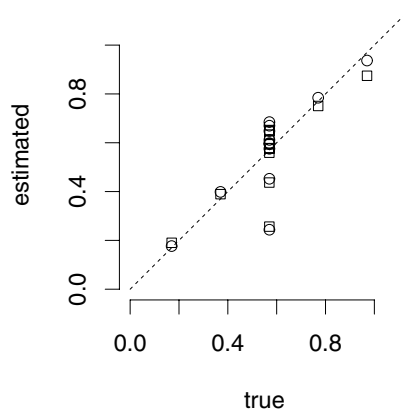

(b) $K^{\text {trans }}$

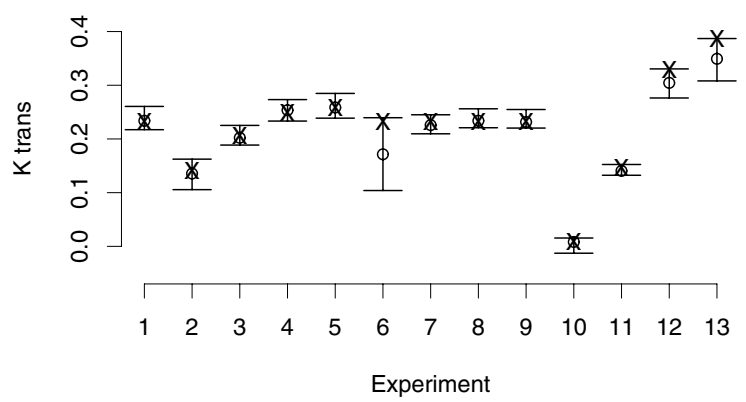

Fig. 2. (a) Scatter plot of true values against estimated values of $F_{p}$ with ad-hoc method (squares) and non-linear fitting (circles). (b) Estimates of $K^{\text {trans }}$ (circles) with $95 \%$ intervals; true values are marked as cross.

\subsection{In Vivo Validation}

The clinical data used in this study were provided by the Paul Strickland Scanner Centre at Mount Vernon Hospital, Northwood, UK. The data were derived from six patients with breast tumor scanned with a $1.5 \mathrm{~T}$ Siemens MAGNETOM Symphony scanner, $\mathrm{TR}=11 \mathrm{~ms}$ and $\mathrm{TE}=4.7 \mathrm{~ms} ; 40$ scans with four sequential slices were acquired in about 8 minutes. A dose of $\mathrm{D}=0.1 \mathrm{mmol} / \mathrm{kg}$ body weight Gd-DTPA was injected at the start of the fifth acquisition using a power injector. One slice from the pre-treatment scan of each patient was used for analysis. Data from this study have been previously reported [13, 3].

Figs. 3(a)-(c) show the observed contrast concentration time series from three voxels (tumor, peri-tumoral rim, normal tissue) together with the fits from (10) and from the proposed semi-parametric approach. It is evident that in all vox-
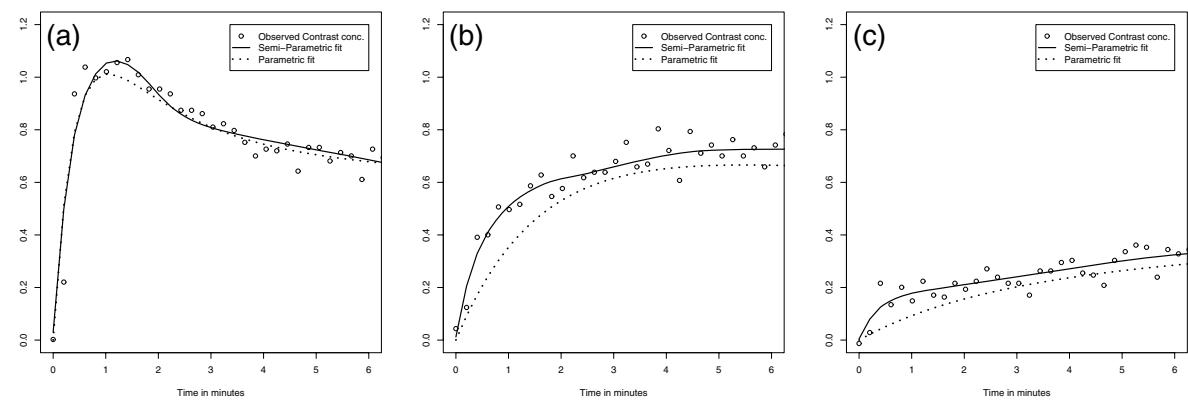

Fig. 3. Observed concentration time series (circles) with fit by parametric (dotted line) and semi-parametric method (solid line) for three voxels: (a) tumor, (b) peri-tumoral rim, (c) normal tissue 
Table 1. Sum of squared error (SQE) between data and fitted function for parametric and semi-parametric method, averaged over all voxels. Last row - Mean percentage of $K^{\text {trans }}$ parameter estimated with semi-parametric approach in respect of values estimated with parametric approach (for tumor voxels)

\begin{tabular}{l||r|r|r|r|r|r} 
Patient & 1 & 2 & 3 & 4 & 5 & 6 \\
\hline SQE, par. & 0.0709 & 0.1267 & 0.3992 & 0.1747 & 0.3432 & 0.1730 \\
SQE, semi-par. & 0.0515 & 0.0614 & 0.1054 & 0.0443 & 0.0844 & 0.0876 \\
perc. $K^{\text {trans }}$ & $195 \%$ & $118 \%$ & $564 \%$ & $801 \%$ & $297 \%$ & $142 \%$
\end{tabular}

els the semi-parametric method fits the data much better than the parametric approach. This is to be expected since the degrees of freedom of the model in the semi-parametric approach are much higher. The figures also indicate that the standard parametric model is not always appropriate for the observed data, where the initial upslope is difficult to fit using the parametric model.

Finally, Table 1 shows the goodness of fit for the data from all six patients and the mean percentage of $K^{\text {trans }}$ obtained from the semi-parametric and the parametric approach. As with the simulated data, the fit is clearly better with the semi-parametric technique. Also, $K^{\text {trans }}$ values are again higher with the semi-parametric method, showing a similar trend as the simulation study.

\section{Conclusion}

In this paper, we have presented a Bayesian P-spline model for quantifying contrast agent concentration time series of DCE-MRI scans. Compared with parametric PK models, the proposed semi-parametric technique provides a much better fit to the data. In particular the proposed method fits the important upslope of the time series more precisely. Parametric non-linear models are typically difficult to estimate due to the convergence issues and problems in specifying consistent starting values. Bayesian non-linear regression can overcome convergence issues, but with an increase in computation time 3. The proposed semi-parametric method provides a reliable, practical algorithm by effectively circumventing the above problems.

In contrast to classical approaches [6, Bayesian P-splines allow the simultaneously estimation of model and smoothing parameters. Adaptive smoothing parameters can easily be obtained, and are important to model the sharp changes in the dynamic series.

The proposed method allows a direct assessment of the response function, i.e., the actual flow in the tissue. Parameters of interest may be estimated by an ad-hoc technique (maximum of response function) or by fitting a non-linear model to the response function. In the latter case, smoothing via P-splines provides error reduction and deconvolution of the arterial input function. Results from simulated data show a clear improvement in parameter estimation. From an initial clinical validation, a similar trend of differences between parameters estimated with parametric and semi-parametric approach was observed. The 
proposed method also provides standard errors for all parameters, and therefore the reliability of the results can be quantified.

\section{Acknowledgements}

Support for Volker Schmid was financed through a research grant from GlaxoSmithKline. We are grateful to David Buckley for providing the simulated data. The clinical data was graciously provided by Anwar Padhani and Jane Taylor.

\section{References}

1. Parker, G.J.M., Padhani, A.R.: $T_{1}$-w DCE-MRI: $T_{1}$-weighted dynamic contrastenhanced MRI. In Tofts, P., ed.: Quantitative MRI of the Brain. Wiley, Chichester, England (2003) 341-364

2. Larsson, H.B., Tofts, P.S.: Measurement of the blood-brain barrier permeability and leakage space using dynamic Gd-DTPA scanning - a comparison of methods. Magnetic Resonance in Medicine 24 (1992) 174-176

3. Schmid, V.J., et al. : Statistical analysis of pharmacokinetical models in dynamic contrast-enhanced magnetic resonance imaging. In Duncan, J., Gerig, G., eds.: MICCAI 2005. (2005) 886-893

4. Twellmann, T., Lichte, O., Nattkemper, T.W.: An adaptive tissue characterization network for model-free visualization of dynamic contrast-enhanced magnetic resonance image data. IEEE Transactions on Medical Imaging 24 (2005) 1256-66

5. Gunn, R.N., Gunn, S.R., Turkheimer, F.E., Aston, J.A.D., Cunningham, V.J.: Positron emission tomography compartmental models: A basis pursuit strategy for kinetic modeling. J Cereb Blood Flow Metab 22 (2002) 1425-1439

6. Jerosch-Herold, M., Swingen, C., Seethamraju, R.: Myocardial blood flow quantification with MRI by model-independent deconvolution. Medical Physics 29 (2002) 886-897

7. Eilers, P., Marx, B.: Flexible smoothing with B-splines and penalties (with comments and rejoinder). Statistical Science 11 (1996) 89-121

8. Lang, S., Brezger, A.: Bayesian P-splines. Journal of Computational and Graphical Statistics 13 (2004) 183-212

9. Tofts, P., Kermode, A.: Measurement of the blood-brain barrier permeability and leakage space using dynamic MR imaging-1. Fundamental concepts. Magnetic Resonance in Medicine 17 (1991) 357-367

10. Gilks, W.R., Richardson, S., Spiegelhalter, D.J., eds.: Markov Chain Monte Carlo in Practice. Chapman \& Hall, London (1996)

11. St.Lawrence, K.S., Lee, T.Y.: An adiabatic approximation to the tissue homogeneity model for water exchange in the brain: I. Theoratical derivation. Journal of Cerebral Blood Flow \& Metabolism 18 (1998) 1365-1377

12. Buckley, D.L.: Uncertainty in the analysis of tracer kinetics using dynamic contrastenhanced $T_{1}$-weighted MRI. Magnetic Resonance in Medicine 47 (2002) 601-6

13. Ah-See, M.L.W., et al.: Does vascular imaging with MRI predict response to neoadjuvant chemotherapy in primary breast cancer? Journal of Clinical Oncology (Meeting Abstracts) 22 (2004) 582 